\title{
High quality ITO thin films grown by de and RF sputtering without oxygen
}

\author{
Ocal Tuna $^{1}$, Yusuf Selamet ${ }^{1}$, Gulnur Aygun $^{1}$ and Lutfi Ozyuzer ${ }^{1,2,3}$ \\ ${ }^{1}$ Department of Physics, Izmir Institute of Technology, TR-35430, Urla, Izmir, Turkey \\ ${ }^{2}$ Teknoma Technological Materials Ltd., IYTE Campus, TR-35430, Urla, Izmir, Turkey \\ E-mail: ozyuzer@iyte.edu.tr (Lutfi Ozyuzer)
}

Received 1 August 2009, in final form 10 December 2009

Published 21 January 2010

Online at stacks.iop.org/JPhysD/43/055402

\begin{abstract}
High quality indium tin oxide (ITO) thin films were grown without oxygen by both dc and RF magnetron sputtering techniques on glass substrates. The effects of substrate temperature, film thickness and sputtering method on the structural, electrical and optical properties of the as-grown films were investigated. The results showed that the substrate temperature had substantial effects on the film properties, in particular on the crystallization and resistivity. When the substrate temperature was increased to $150^{\circ} \mathrm{C}$, crystallization in the (22) plane started appearing for both dc and RF sputtered films. We additionally found that with further increments of substrate temperature, the preferred crystallization orientation changed differently for dc and RF sputtered films. Optical transmission in the visible region for a film thickness of $70 \mathrm{~nm}$ was found to be above $85 \%$. The bandgap was calculated to be about $3.64 \mathrm{eV}$ for the substrate temperature of $150{ }^{\circ} \mathrm{C}$ for a $70 \mathrm{~nm}$ thick film. The value of the bandgap increased with respect to the increment in film thickness as well as substrate temperature. We also measured the temperature dependence of the resistivity and Hall coefficient of the films, and calculated the carrier concentration and Hall mobility. Very low room temperature resistivities for dc and RF magnetron sputtered grown films of about $1.28 \times 10^{-4} \Omega \mathrm{cm}$ and $1.29 \times 10^{-4} \Omega \mathrm{cm}$, respectively, were obtained.
\end{abstract}

\section{Introduction}

Transparent conducting oxide (TCO) thin films, such as zinc oxide, tin oxide, indium tin oxide, cadmium oxide and cadmium tin oxide, are used for a wide range of important applications, such as transparent electrodes in LCDs, organic light emitting diodes, solar cells, plasma display panels, transparent heat reflecting windows as well as surface heaters for cameras, lenses, mirrors, car windows, gas sensors and ohmic contacts for surface-emitting diodes [1-10]. Among the TCO thin films, indium oxide doped with tin (ITO) is widely used due to its unique electrical and optical properties such as good conductivity (about $10^{4} \Omega^{-1} \mathrm{~cm}^{-1}$ ) and high transmittance $(\sim 85 \%)$ in the visible region due to its large bandgap of about $3.70 \mathrm{eV}$. As-grown ITO thin films are usually highly degenerate n-type semiconductors due to their large number of oxygen vacancies as well as substitutional Sn dopants [11-13]. Various growth conditions have the effect

\footnotetext{
3 Author to whom any correspondence should be addressed.
}

of increment in the carrier concentrations contributing to the enlargement of the bandgap, which is known as the BursteinMoss shift $[2,11]$. In addition to the carrier concentration, growth conditions strongly affect the crystallinity, impurity levels and surface roughness of the grown films.

Various techniques, such as electron beam evaporation [14], ion beam assisted deposition [15], pulsed laser ablation [16, 17], ion implantation [18], and dc/RF magnetron sputtering (DCMS/RFMS) [19-21], are used for the growth of ITO thin films. In the majority of reports, $\mathrm{O}_{2}$ is used as a reactive gas during growth and/or post-growth annealing to decrease the resistivity of the films [11, 19, 22]. Nevertheless, oxygen usage during growth and/or post-growth annealing complicates the process and puts an extra strain in vacuum chambers. In the variable range of the In to $\mathrm{Sn}$ ratio used with the sputtering technique, indium-tin alloy with a $(90: 10)$ of In : Sn weight ratio is known to have yielded films with better electrical characteristics [22].

Detailed investigation of RF and dc sputter grown ITO at a single substrate temperature of $300^{\circ} \mathrm{C}$ was done by Qiao 
and Mergel [23]. They used very high powers of above $100 \mathrm{~W}$ yielding very thick films when compared with our samples and derived electrical properties of grown films from optical measurements. However, the optically measured carrier concentration and mobility usually differ from those obtained by electrical measurements. This difference can be explained by a microstructure with pronounced grain boundaries which impedes the dc conductivity [24].

In this study, we investigated the effect of substrate temperature, film thickness and sputtering method on the structural, electrical and optical properties of $10 \mathrm{wt} \%$ tin doped ITO films grown in the same chamber by the DCMS and RFMS methods. In order to simplify the growth process, we did not introduce oxygen during either the growth or the postgrowth annealing processes of the films. The properties of ITO thin films grown by RFMS and DCMS under similar growth conditions were compared. Temperature dependent Hall effect measurements were made to obtain the carrier concentration and mobility of our samples. Very low room temperature resistivities recorded with these samples raise the question of the necessity of additional oxygen usage for high quality ITO thin film growth. Even though we limit the number of films reported for comparison purposes here, high quality ITO thin film growth without added oxygen was highly reproducible.

\section{Experimental procedure}

ITO thin films were deposited on $1.0 \mathrm{~mm}$ thick microscope glass slides by the DCMS and RFMS techniques using an ITO target with a purity of $99.99 \%$ and a weight per cent of 10 to 90 for $\mathrm{SnO}_{2}$ and $\mathrm{In}_{2} \mathrm{O}_{3}$, respectively. The sputtering system [25] has four 2 inch heads (AJA Int.) with dc (Advanced Energy, MDX 1K) and RF (Huttinger, PFG 600RF) power supplies. The target to substrate distance was kept constant at $7.5 \mathrm{~cm}$ for each deposition. An IR heater was used for substrate heating from 150 to $400^{\circ} \mathrm{C}$. The temperature was obtained by a thermocouple on the substrate surface and was controllable with $1 \%$ measurement accuracy. Prior to the growth process, the glass substrates were cleaned each for $10 \mathrm{~min}$ in an ultrasonic bath with acetone, alcohol and deionized water, respectively, and then blown dry with high purity nitrogen gas. The system was evacuated to a base pressure of less than $2 \times 10^{-6}$ Torr prior to the growth. Ar gas flow was adjusted with an MKS mass flow controller maintaining the working gas pressure of about $0.55 \mathrm{~m}$ Torr during deposition. Powers of $15 \mathrm{~W}$ and $40 \mathrm{~W}$ were used for the dc and RF growth processes, respectively. The films were grown at substrate temperatures $\left(T_{\mathrm{s}}\right)$ ranging from 150 to $400^{\circ} \mathrm{C}$ with a $50{ }^{\circ} \mathrm{C}$ increment. The growth rates were $7.7 \pm 0.1 \mathrm{~nm} \mathrm{~min}^{-1}$ and $4.1 \pm 0.1 \mathrm{~nm} \mathrm{~min}^{-1}$ for the dc and RF sputtered films, respectively. ITO films with thicknesses of $40 \pm 1$ and $70 \pm 1 \mathrm{~nm}$ were grown.

The structural characterization of the ITO films was carried out by an X-ray diffractometer (XRD) (Philips X'Pert Pro) with $\mathrm{Cu} \mathrm{K}_{\alpha}$ radiation $(\lambda=0.15406 \mathrm{~nm})$. The optical properties of the ITO thin films were measured by a spectroscopic ellipsometer (SENTECH SE 801) in the $300-850 \mathrm{~nm}$ wavelength range. Photolithography and

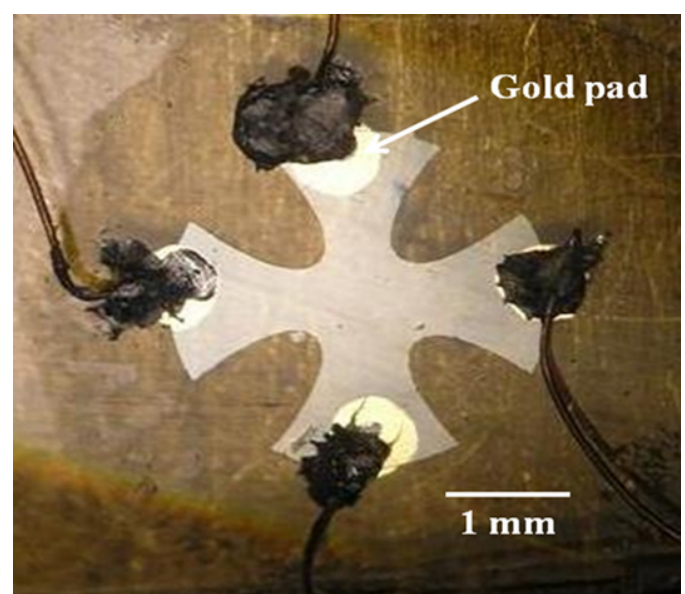

Figure 1. Optical photograph of photolithographically patterned configuration used for electrical measurements. The gold pads can be seen underneath the contacts. The picture was taken while the sample is on the cold finger of the cryostat.

(This figure is in colour only in the electronic version)

ion beam etching techniques were used for patterning the films to a cloverleaf shape which was used to perform the electrical measurements (figure 1). $20 \mathrm{~nm}$ thick gold films were deposited at the corners of the patterned samples by thermal evaporation to avoid electrical contact problems on the patterned films. Wiring onto the gold pads was done by high purity $\mathrm{In}$ and thin $\mathrm{Cu}$ wires. A Labview program controlled set-up consisting of a Keithley 220 Programmable Current Source, a 2182 Nanovoltmeter, a Hall effect card and a temperature controller was used to conduct the electrical measurements by means of the van der Pauw method. The estimated measurement accuracy of resistivity was better than $0.05 \times 10^{-4} \Omega \mathrm{cm}$. The Hall effect measurements under a constant magnetic field of $320 \mathrm{G}$ were done in a liquid $\mathrm{N}_{2}$ cooled cryostat (Janis, ST-100). The thicknesses of the films were measured by an atomic force microscope (Digital Instruments, Multimode SPM, Nanoscope-IV).

Although all films were characterized by the techniques given above, only the critical data will be presented in this paper.

\section{Results and discussion}

The analysis of XRD patterns demonstrate that ITO thin films grown at the substrate temperatures lower than $150^{\circ} \mathrm{C}$ were amorphous. Crystallization was detected in the (22) plane for the substrate temperatures higher than $150{ }^{\circ} \mathrm{C}$ up to $300^{\circ} \mathrm{C}$ for both dc and RF sputtered films (figure 2). Since indium has a face-centred tetragonal crystal structure, the [1 111 l direction is the most densely packed and, consequently, (111) is the lowest energy plane [26]. When the substrate temperature was increased above $300^{\circ} \mathrm{C}$, the crystalline planes changed slowly from (222) to (400) for the DCMS samples and the (440) peak became more apparent. Additionally, the intensity of the peaks also changed from (222) to (440) with respect to the substrate temperature increment for the RFMS samples. For the RFMS samples the (211) and (440) peaks also started 


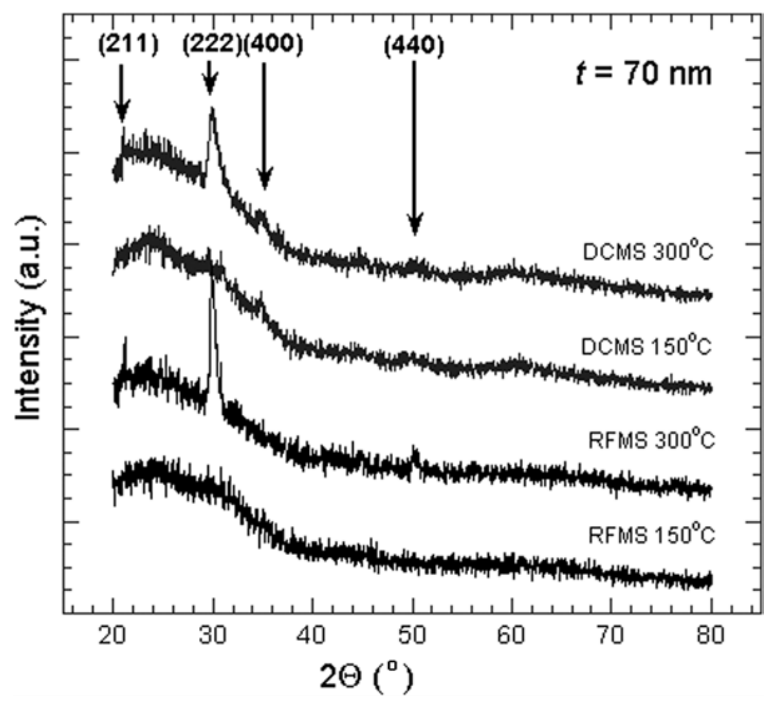

Figure 2. X-ray diffraction patterns of $70 \mathrm{~nm}$ thick ITO films grown by DCMS and RFMS at various substrate temperatures $\left(T_{\mathrm{s}}\right.$ is the substrate temperature and $t$ is the film thickness).

appearing at higher substrate temperatures. Qiao and Mergel reported similar crystallinity dependences on RF and dc power for magnetron sputtered ITO films at $300^{\circ} \mathrm{C}$ [23]. In their study, the changes reported in crystallization orientation with respect to increasing power were quite similar to the changes reported here with our substrate temperatures. The observed similarities can be explained by the statement of Kumar and Mansingh, namely, that if the samples are grown by the bombardment of more energetic particles and/or by particles having high energies when they reached the growth surface, then the $(400)$ orientation is more favoured [27].

Grains of various sizes result in ITO films with a columnar structure in which each grain has close but different growth rate from its own growth orientation which usually remains the same throughout the growth process within the grain [28]. In the light of this argument, only the growth parameters would be responsible for the preferred orientation changes of the grown film on amorphous glass substrates. The data from the literature and the data presented here suggest that substrate temperature increment is vitally important for the increased crystallinity of the films. Since the only growth parameter we varied was the substrate temperature, it must be responsible for these crystallinity plane changes within each growth method. In addition to substrate temperature increments, our films were grown without oxygen; therefore, secondary effects of oxygen vacancies might also be playing a role in these crystal orientation changes. The number of oxygen vacancies, in fact, strongly depends on the substrate temperature. Moreover, increased mobility of the adatoms on the growth surface resulting from the increased substrate temperature should also be considered. Since our growth process was completed in a vacuum environment with no added oxygen, we can expect an increased number of oxygen vacancies in our films grown at elevated substrate temperatures. Indeed, we came across publications that report the changes in preferred orientations from $(222)$ to $(400)$ by reduction in oxygen partial pressure during the growth process [26].

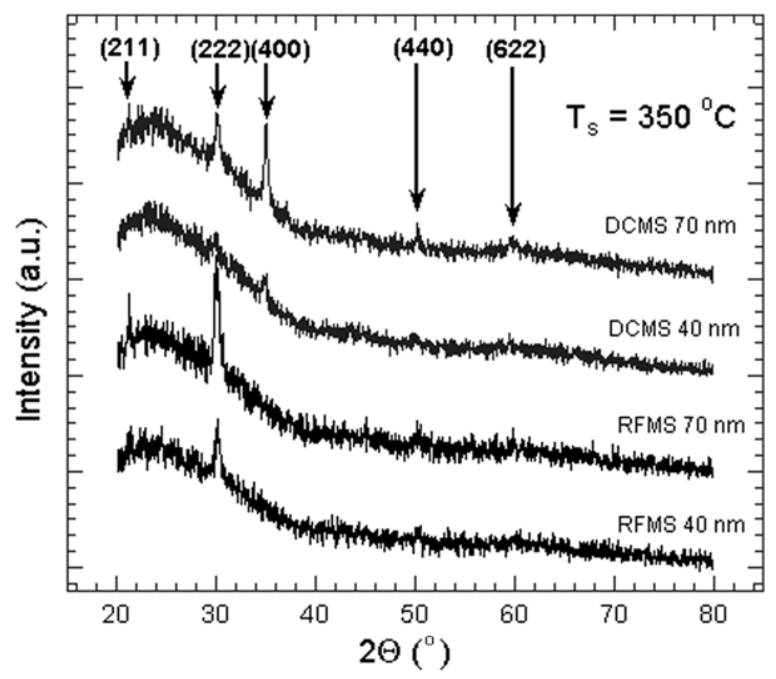

Figure 3. X-ray diffraction patterns of 40 and $70 \mathrm{~nm}$ thick ITO films grown at a substrate temperature of $350^{\circ} \mathrm{C}$.

Similar changes in preferred crystallization planes as a result of increased substrate temperatures were also detected and reported as a result of increment in the mobility of the adatoms and clusters by Nisha et al [11] and Chen et al [26].

Figure 3 shows a comparison of the XRD scans for RF and dc sputtered ITO thin films with thicknesses of 40 and $70 \mathrm{~nm}$ grown at a substrate temperature of $350{ }^{\circ} \mathrm{C}$. Since the optimum characteristics in terms of resistivity for grown films were obtained at this substrate temperature, it was chosen to be a representative for many XRD curves taken. It is clear that increased film thicknesses for both techniques yield more distinguishable XRD peaks. Similar observations, i.e. as the thickness of the ITO film is increased, the crystallinity degree of the films is more pronounced, were reported by Lee et al [29].

The interplanar spacing in the [2 22] direction, $d_{222}$, was calculated. The results were higher than that of the well-known powder XRD interplanar spacing of $0.2921 \mathrm{~nm} . d_{222}$ values at $300{ }^{\circ} \mathrm{C}$ were $0.2969 \mathrm{~nm}$ and $0.2972 \mathrm{~nm}$, respectively, for the $\mathrm{dc}$ and RF grown samples. These differences from the powder interplanar spacing value implied the presence of tensile stress in our samples. Observing larger interplanar spacing values in thin films is quite common. These values get closer to the powder values with increased substrate temperatures and/or post-growth thermal treatments.

The effect of substrate temperature on the as-grown film's electrical properties, i.e. resistivity and bandgap, obtained at room temperature, was also investigated (figure 4). We observed that the resistivity decreases as the substrate temperature increases. This is due to the increment in the carrier concentration, crystallization and grain size as a result of substrate temperature increment (table 1). The change in the preferred crystallization orientation might also have a positive contribution to the lowering of resistivity values as a response to the temperature increments. The lowest resistivity was obtained for both growth methods for the substrate temperature of $350^{\circ} \mathrm{C}$ for $70 \mathrm{~nm}$ thick films as $1.28 \times 10^{-4} \Omega \mathrm{cm}$ (DCMS) and $1.29 \times 10^{-4} \Omega \mathrm{cm}$ (RFMS). These values are some of the 

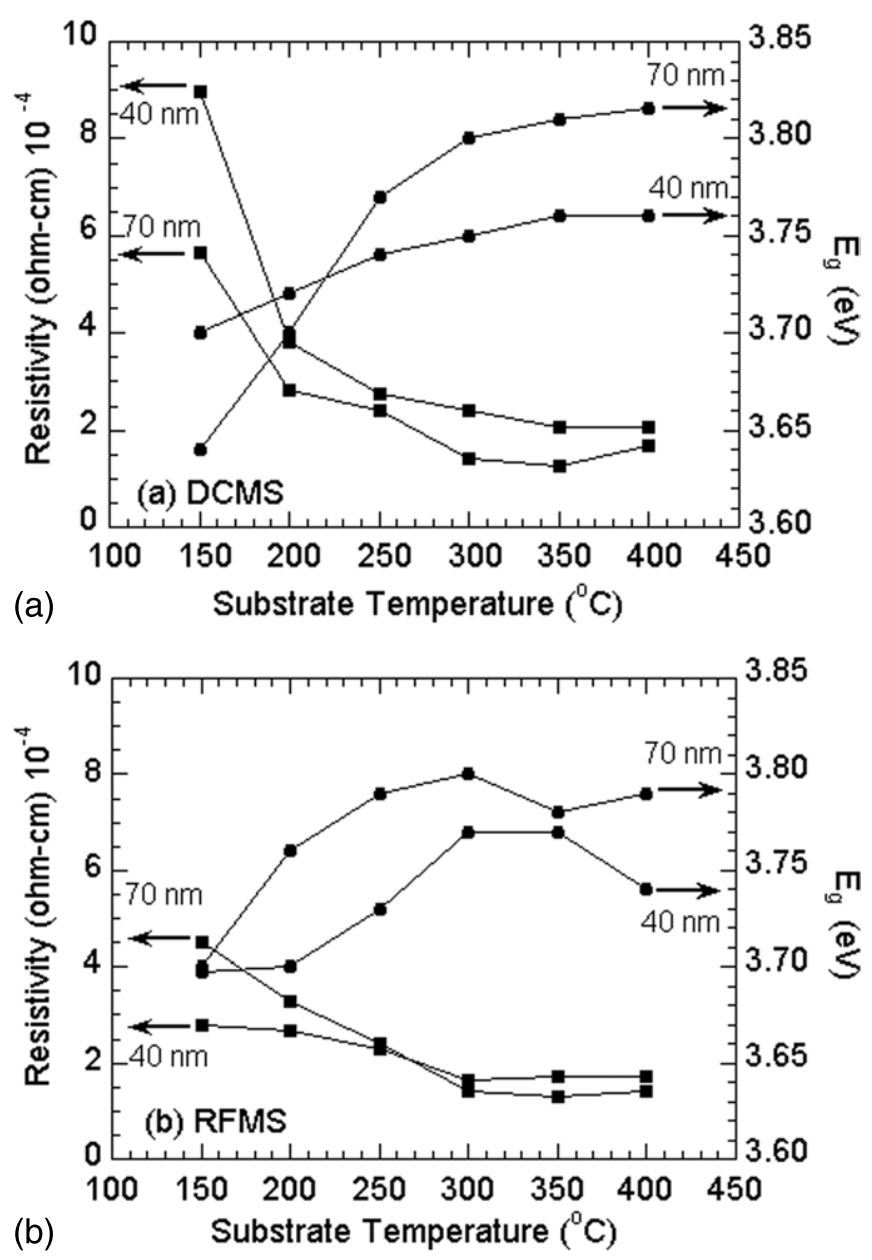

Figure 4. Variation of the resistivity and bandgap as a function of substrate temperature for (a) DCMS and (b) RFMS grown ITO films. Note that the error bars are smaller than the symbol sizes.

lowest measured room temperature resistivities reported for both RF and dc sputtered films [30]. The prominence of the results is clear considering that the films were neither reactively grown nor annealed in any oxidizing atmosphere.

Scherrer's formulation [31] was used for grain size calculations to check whether a correlation exists between grain size and resistivity. Our calculations showed that the resistivities were inversely proportional to the grain sizes of the DCMS grown films (table 1) referring to a correlation between the two mentioned parameters, i.e. grain size and resistivity [32]. When compared with the degree of correlation of grain sizes and resistivities at increased substrate temperatures for the DCMS films, however, the degree of correlation did show an extremely small correlation for the RFMS grown samples. This difference observed with the sputtering method might explain the 4.5 and 3 times decrease in resistivities with increment in substrate temperatures for DCMS and RFMS grown samples, respectively. Since the grain size showed the general trend of proportional increment with increased substrate temperatures (table 1), i.e. the number of grains decreased, the scattering probability from grain boundaries with increasing substrate temperature should decrease.

The resistivities for both RFMS and DCMS grown films at $400{ }^{\circ} \mathrm{C}$ were found to be slightly higher than those obtained at $350{ }^{\circ} \mathrm{C}$ (figure 4). The stoichiometry of the deposited films might be adversely affected due to the possibly higher number of oxygen vacancies under the increased substrate temperatures. To be completely certain about the oxidation status of the grown films at higher substrate temperatures, a chemical analysis of the constituent elements on the surface as well as in the depth profile of the film is necessary and, therefore, an X-ray photoelectron spectroscopy (XPS) analysis has been under consideration and will be be reported elsewhere as a future study. As previously reported by Nisha et al, additionally, some alkali ions might probably diffuse into the film structure at relatively higher growth temperatures from the glass substrate resulting in an adverse effect on mobility [11]. Therefore, it is highly preferable to grow films at a substrate temperature close to $350{ }^{\circ} \mathrm{C}$ to attain the optimum electrical properties. The discussion of the observed bandgap change will be given later in the paper.

Figure 5 shows the normalized resistivities with respect to measurement temperature for $70 \mathrm{~nm}$ thick DCMS and RFMS grown films at the substrate temperature of $350^{\circ} \mathrm{C}$. The resistivity versus temperature shows the expected metallic-like behaviour, i.e. slow increment of resistivities with respect to the increment in measurement temperature for all grown films. The resistivity changed from $1.28 \times 10^{-4}$ to $1.16 \times 10^{-4} \Omega \mathrm{cm}$ for $\mathrm{dc}$ and from $1.29 \times 10^{-4}$ to $1.16 \times 10^{-4} \Omega \mathrm{cm}$ for $\mathrm{RF}$ sputtered films at $350^{\circ} \mathrm{C}$ substrate temperature of $70 \mathrm{~nm}$ thick films with respect to temperature decrement from 295 to $78 \mathrm{~K}$ (table 1). The temperature dependence of resistivity for all grown samples showed nearly the same characteristics. We, furthermore, measured the temperature dependence of the Hall coefficient using the van der Pauw configuration. We observed that the Hall coefficient increased slowly with the increment of measurement temperature from 80 to $300 \mathrm{~K}$. This is in agreement with ITO thin films being highly degenerate of n-type semiconductors.

The room temperature carrier concentrations were calculated using the inverse relation of the measured Hall coefficient with the carrier concentration for both DCMS and RFMS grown samples. It can be clearly observed from figure 6 that the carrier concentration increases monotonically with increasing substrate temperature. Similar behaviour was also reported in other studies for elevated substrate [33] and/or annealing temperatures [34]. It is well known that oxygen vacancies and $\mathrm{Sn}$ activation in the In site are the two main sources of free electrons in ITO thin films. As discussed above, the crystallinity of the ITO films increases with increased substrate temperature as well. Higher degree of crystallinity would mean that the smaller number of defects act as traps, i.e. scattering centres. We also expected to have a larger number of oxygen vacancies with increasing substrate temperatures, contributing to the n-type behaviour. The observation of preferred crystallization orientation in (400) with increasing substrate temperature also suggests that the growth proceeds towards the oxygen deficient sides. The observed increment in carrier concentration as a result of increasing substrate temperature might also be due to an increment in the diffusion of tin atoms with four valence electrons into the interstitial locations and grain boundaries, as suggested earlier by Reddy et al [2]. 
Table 1. Properties of the DCMS/RFMS grown $70 \mathrm{~nm}$ thick ITO thin films; $T_{\mathrm{s}}$ substrate temperature, $E_{\mathrm{g}}$ bandgap, $\rho$ resistivity, $n$ carrier concentration, $\mu$ Hall mobility, $d$ grain size. Note that the grain size cannot be found for substrate temperatures of $150{ }^{\circ} \mathrm{C}$.

\begin{tabular}{|c|c|c|c|c|c|c|c|c|}
\hline \multirow[b]{2}{*}{ Growth method } & \multirow[b]{2}{*}{ Sample code } & \multirow[b]{2}{*}{$T_{\mathrm{s}}\left({ }^{\circ} \mathrm{C}\right)$} & \multicolumn{2}{|c|}{$\rho\left(10^{-4} \Omega \mathrm{cm}\right)$} & \multirow[b]{2}{*}{$\mathrm{n}\left(\mathrm{cm}^{3} \times 10^{21}\right)$} & \multirow[b]{2}{*}{$\mu\left(\mathrm{cm}^{2} \mathrm{~V}^{-1} \mathrm{~s}^{-1}\right)$} & \multirow[b]{2}{*}{$E_{\mathrm{g}}(\mathrm{eV})$} & \multirow[b]{2}{*}{$\mathrm{d}(\mathrm{nm})$} \\
\hline & & & $300 \mathrm{~K}$ & $78 \mathrm{~K}$ & & & & \\
\hline DCMS & ITO1 & 150 & 2.67 & 5.64 & 0.43 & 53.9 & 3.64 & - \\
\hline DCMS & ITO2 & 200 & 2.53 & 2.66 & 0.60 & 40.0 & 3.70 & 6.4 \\
\hline DCMS & ITO3 & 250 & 2.39 & 2.23 & 0.81 & 31.9 & 3.77 & 6.8 \\
\hline DCMS & ITO4 & 300 & 1.42 & 2.29 & 1.39 & 31.4 & 3.80 & 7.5 \\
\hline DCMS & ITO5 & 350 & 1.28 & 1.16 & 1.89 & 25.7 & 3.81 & 16.6 \\
\hline DCMS & ITO6 & 400 & 1.68 & 1.53 & 2.00 & 18.5 & 3.81 & 19.5 \\
\hline RFMS & ITO1 & 150 & 4.49 & 4.04 & 0.51 & 25.2 & 3.70 & - \\
\hline RFMS & ITO2 & 200 & 3.29 & 3.01 & 0.74 & 25.2 & 3.76 & 15.4 \\
\hline RFMS & ITO3 & 250 & 2.40 & 2.20 & 1.18 & 21.9 & 3.79 & 17.0 \\
\hline RFMS & ITO4 & 300 & 1.40 & 1.81 & 1.30 & 30.0 & 3.80 & 17.1 \\
\hline RFMS & ITO5 & 350 & 1.29 & 1.15 & 1.46 & 32.7 & 3.78 & 16.0 \\
\hline RFMS & ITO6 & 400 & 1.40 & 1.27 & 1.93 & 23.0 & 3.79 & 17.8 \\
\hline
\end{tabular}

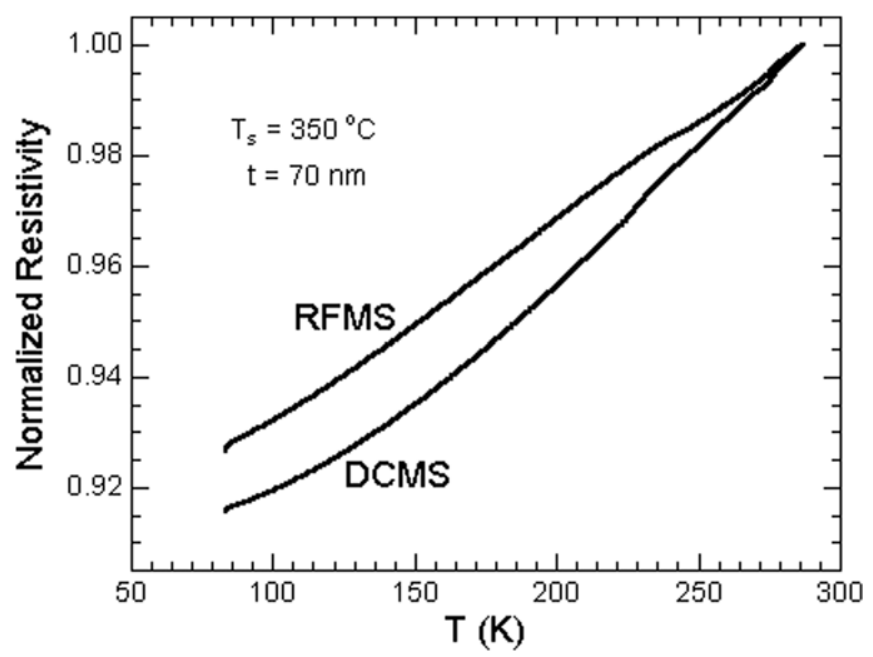

Figure 5. Temperature dependence of electrical resistivity normalized with respect to the measured values of $286 \mathrm{~K}$ of ITO thin film.

Figure 6(a) shows that as the substrate temperature increases, the Hall mobility of the ITO thin films consistently decreases for the DCMS samples. Even though carrier concentration with respect to substrate temperature shows similar characteristics for the DCMS and RFMS grown samples, mobility exhibits different behaviour for DCMS from that of RFMS grown samples with respect to increasing substrate temperatures (figure 6(b)). Mobility decreased for the DCMS grown samples with increasing substrate temperature as expected. The inverse relationship between mobility and carrier concentration suggests that the ionized impurity scattering was the dominant mechanism. Mobility for RFMS grown samples, however, showed scattered data with respect to substrate temperature increments. The mobility values for the RFMS grown samples were comparatively lower than those of the DCMS grown samples. Contrary to the grain size increment, measured mobility values decrease with increasing substrate temperature for DCMS grown samples. This observed phenomenon can be explained by means of other effective scattering mechanisms such as ionized impurity scattering. For the DCMS grown samples, in fact, mobility
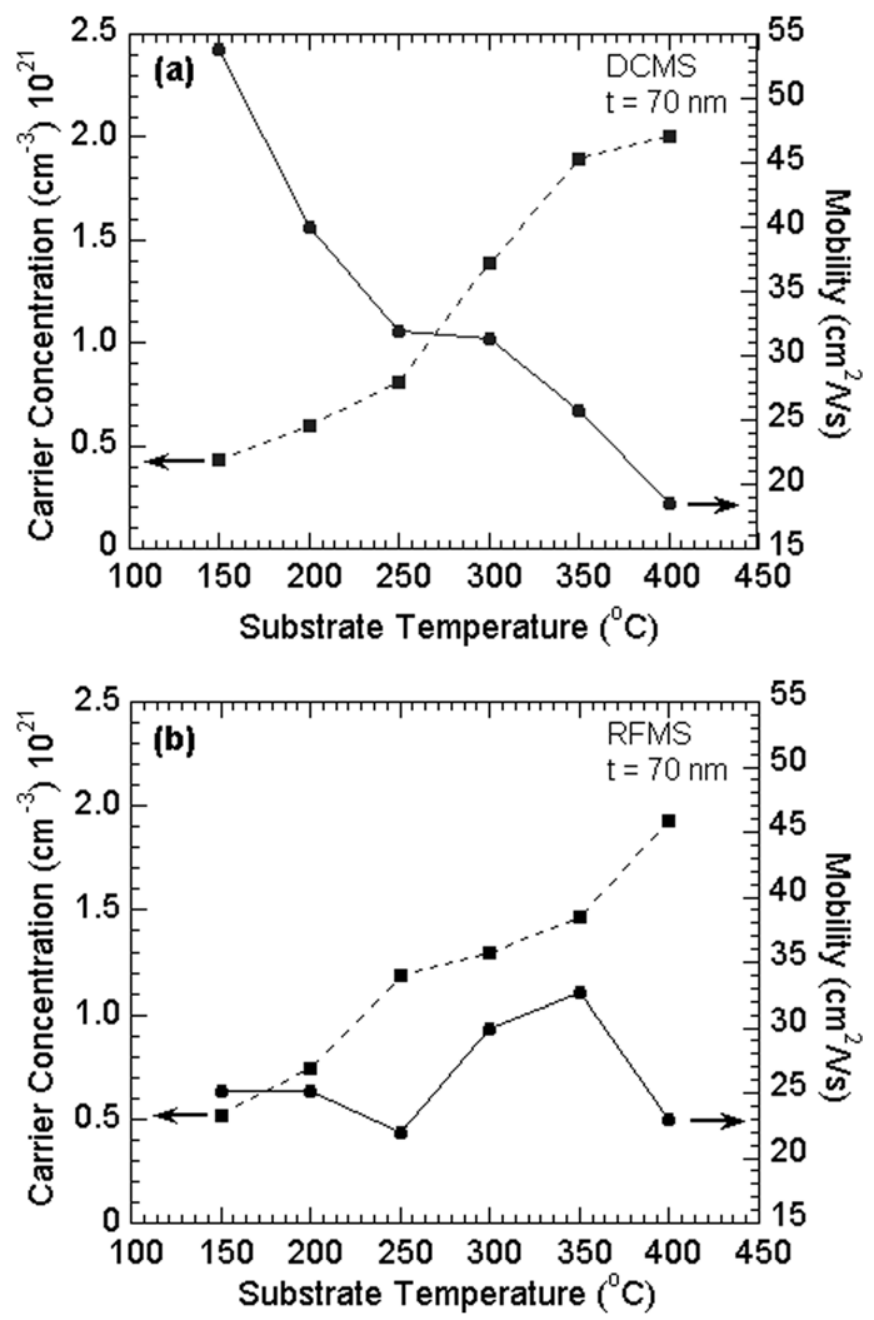

Figure 6. Substrate temperature dependence of room temperature carrier concentrations and Hall mobilities of $70 \mathrm{~nm}$ thick ITO thin films grown with (a) DCMS and (b) RFMS.

versus carrier concentration behaviour in logarithmic scale for both parameters showed a regression line with a power of -0.54 , which is close to the well-known theoretical prediction of $-2 / 3$ power dependence for ionized impurities. On the 
other side, the same plot for the RFMS grown samples did not show any particular relationship with temperature, suggesting that several scattering mechanisms were in effect for different substrate temperature ranges.

For the RFMS grown ITO films, the increase in mobility for temperatures higher than $250^{\circ} \mathrm{C}$ can be attributed to the higher degree of crystallinity, resulting in less grain boundary scattering. The increase in crystallinity with substrate temperature was observed from XRD data analysis, as discussed above. However, above $350^{\circ} \mathrm{C}$, the avalanched number of carrier concentration limits the mobility and, as a result, mobility rapidly decreases. Similarly, mobility decreases very slowly in the $250-300^{\circ} \mathrm{C}$ substrate temperature region for the dc sputtered films. XRD observed tensile stress for our films might have additional adverse effects on the mobility behaviour with respect to substrate temperature increments since it also modifies the band structure.

It intrigued us that the mobility kept decreasing even though the grain size increment was tripled for the DCMS grown samples. In order to get further information, we calculated electron mean free paths using the relation of $l=$ $h / 2 q(3 n / \pi)^{1 / 3} \mu$ [13] for all of our samples, and obtained a range from 20 to $39 \mathrm{~nm}$, all being larger than the grain sizes. This led us to conclude that the grain boundary scattering should also not be discarded. Relatively larger grain sizes for DCMS compared with RFMS grown samples make the ionized impurity scattering mechanism dominant in the DCMS samples. In contrast, both ionized impurities and grain boundary scatterings were dominant in the RFMS grown samples leading to scattered data with respect to substrate temperature increments.

Optical transmissions of the ITO thin films were measured by a spectroscopic ellipsometer in the wavelength range 300$850 \mathrm{~nm}$ (insets of figure 7). $70 \mathrm{~nm}$ thick ITO films were $80-85 \%$ transparent in the visible region. High transmission in the visible region reflects the large bandgap of thin ITO films (figures $4(a)$ and $(b)$ ). The fact that the bandgap of the ITO thin films further increased with increasing substrate temperature is explained with the Burstein-Moss shift [13, 35]. The increasing number of carriers with substrate temperature increments leads to filling of the lowest energy states in the conduction band, thereby causing an enlargement in the bandgap of the ITO films. The fact that lowering the bandgap at the substrate temperature of $300-350{ }^{\circ} \mathrm{C}$ for $70 \mathrm{~nm}$ thick sputtered ITO film might have resulted from the decreasing grain size at higher substrate temperatures (table 1) and, therefore, more scattering from the larger sized grain boundaries is realized.

The absorption coefficient, $\alpha$, versus photon energy plot can be used to find the bandgap energy value, $E_{\mathrm{g}}$, of the ITO thin films (figure 7). Absorption can be calculated using the formula $[19,36]$

$$
T-R=\exp (-\alpha t)
$$

where $T$ and $R$ are the transmittance and reflectance, respectively, and $t$ is the thickness of the film. Since the reflectance of conducting oxide thin films is very low in the
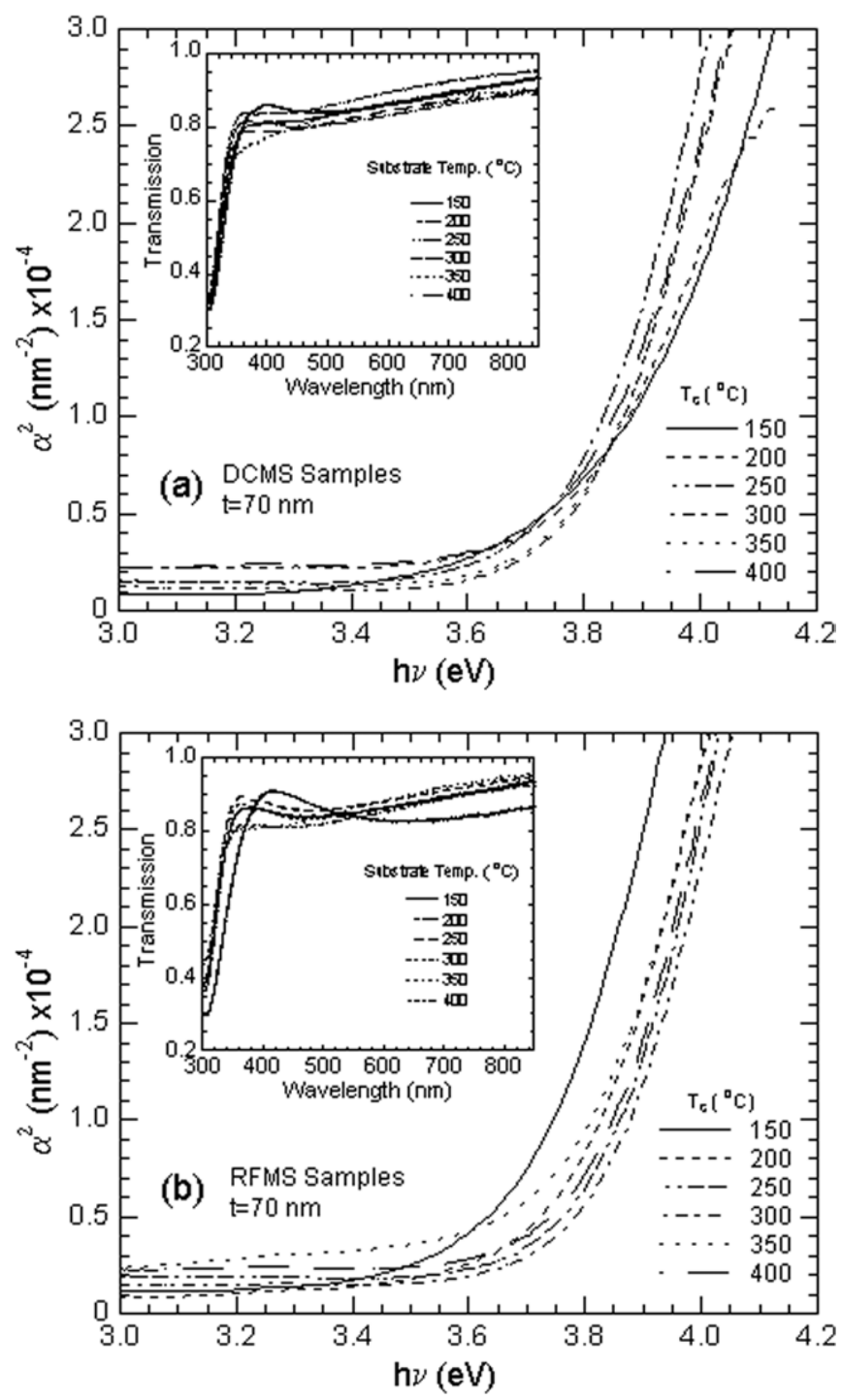

Figure 7. $\alpha^{2}$ versus $h v$ for $70 \mathrm{~nm}$ thick ITO thin film grown by the (a) DCMS and (b) RFMS methods at various substrate temperatures. Insets show transmission spectra of those ITO thin films.

visible and UV range, it can be neglected [19,35], and then the absorption coefficient can be simplified as

$$
\alpha^{2}=\frac{(\ln T)^{2}}{t^{2}} .
$$

Since indium oxide has a direct transition, i.e. $\alpha h v=$ $A\left(h v-E_{\mathrm{g}}\right)^{1 / 2}$, the bandgap of the films can be calculated by extrapolating the linearly increasing portion of the absorption coefficient, $\alpha^{2}$, versus $h v$ curve. It can also be seen from figure 4 that there exists a correlation between bandgap and resistivity, i.e. while the resistivity decreases, the bandgap increases with respect to increased substrate temperature. The fact that the increase in bandgap is related to the increase in carrier concentration with respect to increased substrate temperatures can also be seen in figure 6. This bandgap widening in our ITO films can be very well explained by the Burstein-Moss shift. 


\section{Conclusions}

High quality ITO thin films were grown by both DCMS and RFMS at various substrate temperatures without introducing any oxygen into the growth environment. The growth temperature was shown to play a highly important role since there was a strong correlation between deposition temperature and as-grown film properties such as crystallinity, Hall mobility and electrical resistivity. As previously reported, we also confirmed that the ITO film thickness favourably affects its properties, particularly crystallinity, resistivity and bandgap energy width. The deposited films at the growth substrate temperature of $350^{\circ} \mathrm{C}$ had the lowest room temperature resistivity reported in the literature and the largest bandgap for both DCMS and RFMS methods. The observed preferred crystalline orientations were linked to the increased oxygen vacancies in the grown film with respect to increasing substrate temperature and energetics of the growth method. The grown ITO films showed very low room temperature resistivities and high transmission in the visible region. For the DCMS grown samples the mobility was limited mostly by ionized impurity scattering, whereas for the RFMS ones the mobility was limited by both ionized impurity and grain boundary scatterings. We deduced from our measurements that substrate temperatures of around $300-350{ }^{\circ} \mathrm{C}$ were effective in growing ITO thin films with good electrical properties in terms of resistivity, bandgap and carrier concentration for both DCMS and RFMS methods.

\section{Acknowledgments}

This research is partially supported by the Ministry of Industry of Turkey, SANTEZ project number 00058.STZ.2007-1 and the Scientific and Technical Research Council of Turkey (TUBITAK) project number 107T117. LO acknowledges support from the Turkish Academy of Sciences, in the framework of the Young Scientist Award Programme (LO/TUBA-GEBIP/2002-1-17).

\section{References}

[1] Tang C W and Vanslyke S A 1987 Appl. Phys. Lett. 51913

[2] Reddy V S, Das K, Dhar A and Ray S K 2006 Semicond. Sci. Technol. 211747
[3] Li G, Chu C W, Shrotriya V, Huang J and Yang Y 2006 Appl. Phys. Lett. 88253503

[4] Whang K W and Kim J K 2005 J. Display Technol. 1295

[5] Brauer G 1999 Surf. Coat. Technol. 112358

[6] Rottmann M and Heckner K H 1995 J. Phys. D: Appl. Phys. 281448

[7] Zhao L, Zhou Z, Peng H and Cui R 2005 Appl. Surf. Sci. 252385

[8] Comini E, Faglia G and Sberveglieri G 2001 Sensors Actuators B 7873

[9] Tang Z, Chan P C H, Sharma R K, Yan G, Hsing I M and Sin J K O 2001 Sensors Actuators B 7939

[10] Matin M A et al 1994 Electron. Lett. 30318

[11] Nisha M, Anusha S, Antony A, Manoj R and Jayaraj M K 2005 Appl. Surf. Sci. 2521430

[12] Akkad F E, Marafi M, Punnoose A and Prabu G 2000 Phys. Status Solidi 177445

[13] Wohlmuth W and Adesida I 2005 Thin Solid Films 479223

[14] Ali H M, Mohamed H A and Mohamed S H 2005 Eur. Phys. J. Appl. Phys. 3187

[15] Liu C, Mihara T, Matsutani T, Asanuma T and Kiuchi M 2003 Solid State Commun. 126509

[16] Adurodija F O, Izumi H, Ishihara T, Yoshioka H, Yamada K, Matsui H and Motoyama M 1999 Thin Solid Films 35079

[17] Adurodija F O, Bruning R, Asia I O, Izumi H, Ishihara T and Yoshioka H 2005 Appl. Phys. A $\mathbf{8 1} 953$

[18] Sawada M and Higuchi M 1998 Thin Solid Films 317157

[19] Lee H C and Park O O 2004 Vacuum 75275

[20] Kerkache L, Layadi A, Dogheche E and Remiens D 2006 J. Phys. D: Appl. Phys. 39184

[21] Deng W, Ohgi T, Nejo H and Fujita D 2001 Appl. Phys. A 72595

[22] Adurodija F O, Izumi H, Ishihara T, Yoshioka H, Motoyama M and Murai K 2000 J. Vac. Sci. Technol. A 18814

[23] Qiao Z and Mergel D 2005 Thin Solid Films 484146

[24] Mergel D and Qiao Z 2004 J. Appl. Phys. 955608

[25] Ulucan S, Ozyuzer L and Okur S 2007 J. Optoelectron. Adv. Mater. 9449

[26] Chen Y, Zhou Y, Zhang Q, Zhu M and Liu F 2007 J. Mater Sci.: Mater. Electron. 18411

[27] Kumar C and Mansingh A 1989 J. Appl. Phys. 651270

[28] Jung Y S and Lee S S 2003 J. Cryst. Growth 259343

[29] Lee H C, Seo J Y, Choi Y W and Lee D W 2003 Vacuum 72269

[30] Stowell M, Muller J, Ruske M, Lutz M and Linz T 2007 Thin Solid Films $\mathbf{5 1 5} 7654$

[31] Scherrer P 1918 Göttinger Nachr. 298

[32] Lee H C and Park O O 2006 Vacuum 80880

[33] Meng L J and Santos M P D 1998 Thin Solid Films 32256

[34] Guillen C and Herrero J 2007 J. Appl. Phys. 101073514

[35] Zhang K, Zhu F, Huan C H A and Wee A T S 2000 Thin Solid Films 376255

[36] Sanon G, Rup R and Mansingh A 1991 Phys. Rev. B 445672 